\title{
Thickness characterization of multi-layer coated steel by terahertz time-of-flight tomography
}

Min Zhai, ${ }^{1,2}$ Alexandre Locquet, ${ }^{1,2}$ Cyrielle Roquelet, ${ }^{3}$ L. Agrizzi Ronqueti, ${ }^{3}$ and D.S. Citrin ${ }^{1,2^{*}}$

${ }^{1}$ Georgia Tech-CNRS UMI2958, Georgia Tech Lorraine, 2 Rue Marconi, 57070 Metz, France

${ }^{2}$ School of Electrical and Computer Engineering, Georgia Institute of Technology, Atlanta, Georgia 30332-0250 USA

${ }^{3}$ ArcelorMittal Maizières Research SA, Voie Romaine, 57280 Maizièreslès-Metz, France

*Corresponding author: david.citrin@ece.gatech.edu Tel: +1 404 717-3085 


\section{ABSTRACT}

Terahertz time-of-flight tomography is employed to characterize the individual layers in a multilayer coating on steel substrates. The multilayers, containing up to three layers, consist first of a cataphoretic layer, sealer, and varnish progressing upward from the steel substrate. The individual layer thicknesses were reconstructed from the experimental data using autoregressive spectral extrapolation based on the modified covariance method (AR/MCM), which extrapolates the frequency components of the transfer function into the low signal-tonoise-ratio (SNR) regions from the high SNR regions. Due to the high sensitivity of $\mathrm{AR} / \mathrm{MCM}$ to the presence of noises, wavelet denoising, in some cases, was needed to precondition the data prior to application of AR/MCM. The method is verified numerically and experimentally, showing that it can reconstruct individual layers in the $10-\mu \mathrm{m}$ range, and good agreement is found between the thicknesses of individual layers extracted by AR/MCM and characterized by eddy-current measurements.

Index Terms - Terahertz imaging; Terahertz time-of-fight-tomography; Multilayered coatings; Deconvolution; Nondestructive testing. 


\section{INTRODUCTION}

To date, the conventional nondestructive methods to measure coating thickness are dominated by magnetic gauges [1], eddy-current techniques [2],[3],[4] and ultrasonic testing [5],[6],[7]. These three techniques, for the most parts, involve direct contact with the sample surface. Moreover, for multilayer coatings, ultrasonic testing, may not provide sufficient resolution to separate those echoes reflected from each interface due to the limitation in operating frequency. Eddy-current techniques and magnetic gauges fail to provide the individual layer thickness and rather identify the sum of total layers on metallic substrate. Moreover, because eddy-current techniques and magnetic gauges could not map the entire surface of sample, therefore, thickness distribution estimation based on limited pixels have unexpected errors compared with its own thickness information. The disadvantages hinder the application of eddy current- and acoustic -based approaches for in-line thickness measurement with a short period.

Approaches with a higher resolution, that can provide a global picture of the individual layer thickness for an entire multilayer coating, in a nondestructive and noncontact fashion, are therefore of considerable interest. Terahertz (THz) time-of-flight (TOF) tomography, as a nondestructive, noncontact, and nonionizing approach, has achieved success for a range of applications, including pharmaceuticals [8],[9], archaeology [10],[11], steel production [12],[13], and security [14],[15]. In particular, THz TOF tomography has been applied to measure individual layers in multilayer paint coatings in Refs. [16],[17]. It should be noted that in these works, determination of the individual layer thicknesses is based on sparse deconvolution (without prior knowledge of the structure of the material) [17] or on extensive training of a machine-learning model (also without prior knowledge of structure of material, but with the material parameters of each individual layers) [16]. The stratigraphy of a layered 
sample subjected to an incident roughly single-cycle $\mathrm{THz}$ pulse can be quantitatively measured based on the time delays between pulses (henceforth echoes) reflected from various internal and external interfaces due to the dielectric discontinuities, and thus the name TOF tomography. When, however, the thickness of an individual layer is optically thin in $\mathrm{THz}$ regime, the echoes reflected from successive interfaces will temporally overlap, rendering the individual echoes to be visually indistinct.

Numerous approaches have been proposed and applied to enhance the ability to reconstruct the stratigraphy of structures with optically thin layers. Frequency-domain filtering (Gaussian [18], double-Gaussian [19],[20], Wiener [20],[21], and Hanning [17],[12]) together with wavelet denoising is a frequently used approach to extract the impulse-response function. Filtering may suppress electrical noises, but also may result in the loss of valuable information when a smaller cutoff frequency value $f_{c}$ is applied. Sparse deconvolution based on an iterative shrinkage algorithm, which assumes a sparse representation of the impulseresponse function, is not simply a filter [22],[23]. Based on this method, the detailed stratigraphy of $17^{\text {th }}$ century easel painting Madonna in Preghiera was successfully reconstructed [23]. Due to the large computational costs when a large volume of data points has to be collected, this method can fail to provide timely information for in-line quality inspection. Another promising deconvolution technique, autoregressive spectral extrapolation (AR), likewise not a filter, is presented in Ref. [24],[25]. This method aims at extrapolating the entire frequency spectrum based on frequency components within high-SNR regions. Based on Ref. [24], a single-layer polymer coating with a thickness of $22.5 \mu \mathrm{m}$ on metallic substrate was resolved successfully. Other methods are also proposed to extract the structural information. Yasui et al. proposed a paintmeter for two-dimensional mapping of the paintingthickness distribution for single- and multiple-layer paint films based on $\mathrm{THz} \mathrm{TOF}$ tomography [26]. Yasuda et al. applied a numerical parameter fitting based on multiple- 
regression analysis with the least-square method to enhance the axis resolution for $\mathrm{THz}$ paint meter [27]. Zeitler's group incorporated the etalon effect into the numerical parameter fitting method. to measure the thickness and quality of materials up to four layers [28]. The coating thickness down to $18 \mu \mathrm{m}$ was resolved by this approach. Krimi [29] and Ellrich [30] designed a robust advanced regression approach with a self-calibration model and successfully reconstructed the stratigraphy of a four-layer coating with minimum individual layer thickness $\sim 5 \mu \mathrm{m}$. THz TOF tomography has been applied to measure individual layers in multilayer paint coatings in Refs. [16] where determination of the individual layer thicknesses is based on extensive training of a machine-learning model [16].

In this study, a set of multilayer coated steel coupons were measured by $\mathrm{THz}$ TOF tomography using a TPS spectra 3000 on a reflection module (TeraView Ltd., Cambridge, U.K.). Individual layer thicknesses are determined based on measurements carried out on the completed multilayer coating.- AR based on the modified covariance method (AR/MCM) to reconstruct the stratigraphy of the multilayer coatings where individual layer thicknesses are in some cases optically thin (in the $10-\mu \mathrm{m}$ range). The results thus obtained are compared with layer thicknesses obtained from eddy-current measurements, though such measurements required interrupting the multilayer deposition between successive layers to make the measurements. Good agreement between AR/MCM and the eddy-current results was found.

\section{PRINCIPLE}

AR is parametric approach that reconstructs an extended frequency band of a signal compared with the limited high signal-to-noise band of the measurement. The extrapolation in frequency space is based on a weighted sum of the previous $p$ points in frequency, where $p$ is called the order of the AR model. One of the first practical application of AR was to retrieve the source wavelet from a seismic signal by Lines and Clayton [31]. 
In the following discussion we clarify how the AR approach works. A block diagram showing the implementation of the algorithm is shown in Fig. 1. In the AR process, assuming the discretized transfer function $H_{i}(i$ indexes the discretized frequency) within a window $f_{i_{L}}<f_{i}<f_{i_{H}}$, where $f_{i_{L}}$ and $f_{i_{H}}$ define the lower- and higher- frequency limits of the window of the band where we have signal-to-noise data, the AR model serves as a prediction filter to find data components outside that window for $i>i_{H}$ using the forward prediction equation

$$
\widehat{H}_{i}=-\sum_{k=1}^{p} a_{k} H_{i-k} \text {. }
$$

Similarity, the backward prediction filters is used to find the missing component for $i<i_{L}$,

$$
\widehat{H}_{i}=-\sum_{k=1}^{p} b_{k} H_{i+k},
$$

where $a_{k}$ and $b_{k}$ are the coefficients of the AR forward and backward prediction filters, respectively.

Selecting an appropriate order $p$ is essential for the success of the AR process. On one hand, a too small value means that the AR model is too simple to represent a signal with many subtle though important features. On the other hand, for an excessively large value of $p$, the model will attempt to fit all features, including spurious features, such as arising from noise. Hence, there is a tradeoff between the accuracy of the predicted results and the sensitivity of the AR model to spurious features in the reflected signal. The optimum forward and backward coefficients are determined by minimizing the squared error $\varepsilon_{p}^{2}$ between the data extrapolated by the AR model and the available data. In this case, the sum of both the forward and backward prediction errors is

$$
\varepsilon_{p}^{2}=\sum_{i=i_{L}}^{N_{B W}-p}\left|\widehat{H}_{i}-\sum_{k=1}^{p} b_{k} H_{i+k}\right|^{2}+\sum_{i=i_{L}+p}^{N_{B W}}\left|\widehat{H}_{i}-\sum_{k=1}^{p} a_{k} H_{i-k}\right|^{2}
$$

where $N_{B W}$ is the number of available data points within the high signal-to-noise bandwidth $\left(N_{B W}=i_{H}-i_{L}+1\right)$ 
Akaike's information theoretic criterion (AIC), based on the information theoretic entropy, offers a relative estimate of the information lost, and is a widely used goodness-of-fit measure [32],[33]. AIC qualifies the compromise between the accuracy and the complexity of the model, and is defined as

$$
\operatorname{AIC}[p]=\operatorname{Nlog}\left(\varepsilon_{p}^{2}\right)+2 p
$$

where $2 p$ is the additive penalty term for increasing order, i.e., for any extra coefficients that do not significantly reduce the modeling error. According to Akaike's theory, the minimum value of AIC corresponds to the best-fit of the AR model.

AR deconvolution based on Burg method (AR/BM) has attracted interest due to its superresolution capability [24],[34]. However, due to the high tendency of line-splitting (reconstructions including artifacts that suggest additional layers that are not present) when a larger order is applied as well as the additional constraint from Levinson-Durbin recursion [24], the AR/BM result may not provide reliable reconstruction of multilayered coatings, especially when the absence of prior knowledge of the various layers since in practice the choice of parameters to provide reliable reconstruction may require one to know the layer thicknesses beforehand, which, needless to say, defeats the entire purpose. 
AR based on the modified covariance method (AR/MCM) to minimize the sum of squares of the forward and backward prediction errors, is employed [34]. Compared with AR/BM, the most remarkable advantage of $\mathrm{AR} / \mathrm{MCM}$ is the reduced occurrence of line-splitting due to the restriction in the AR order selection. A drawback is that the stability of AR/MCM cannot be guaranteed when the poles of the system lie outside the unit circle. In order to avoid these singularities, the solution we proposed to stabilize the AR model is to reflect the poles of the model that are outside the unit circle to the inside of the unit circle by reciprocating the magnitude as

$$
D_{i}^{\prime}= \begin{cases}D_{i} & \left|D_{i}\right| \leq 1 \\ \frac{1}{\left|D_{i}\right|^{2}} D_{i} & \left|D_{i}\right|>1\end{cases}
$$

where $D_{i}$ are the poles of the system, and $D_{i}^{\prime}$ are the updated poles. More details can be found in Ref. [35]. Because the high sensitivity of AR model to SNR, the measured data is preconditioned by wavelet denoising to improve the accuracy of AR/MCM. In contrast to typical frequency-domain filter (typically, low-pass and band-pass), the bandwidth of the impulse response function is not narrowed by wavelet denoising.

\section{SAMPLES \& MEASUREMENT}

\section{Coated samples}

For this study, three sets of coated steel coupons with different layer configurations are studied as depicted in Fig. 2. The dimension of all samples is $10 \mathrm{~cm} \times 20 \mathrm{~cm}$. The first set of samples numbered 5, 9, and 10 have a single cataphoretic layer on a flat steel coupon. The second set numbered 12, 14, and 16 have two layers, viz. the cataphoretic layer and a sealer. The third set numbered 11, 15, and 17 have three layers, namely the cataphoretic layer, a sealer, and lacquer. Each layer performs a different function. The cataphoretic layer, which is applied electrochemically, provides corrosion protection of the metal substrate. The function 
of the sealer is to promote adhesion between the cataphoretic layer and the layer above, and moreover regularize the rough cataphoretic layer to lead to a smoother overall finish. The lacquer provides ultraviolet protection, resistance to mechanical abrasion, and resistance against chemical attack. In our samples, the lacquer is the thickest layer.

\section{Terahertz time-of-flight tomography}

The measurement in this work is employed by a pulsed, broadband $\mathrm{THz}$ time-domain spectroscopy (TDS) system (TeraView TPS Spectra 3000) shown schematically in Fig. 3. The GaAs photoconductive antenna is excited and produce roughly single-cycle THz pulses with bandwidth extending from $60 \mathrm{GHz}$ to $3 \mathrm{THz}$. The laser used is an Er-doped fibre laser which emits $780 \mathrm{~nm}$ pulses with sub-100 femtosecond pulse duration at a repetition rate of $100 \mathrm{MHz}$ and has an average output power of $>65 \mathrm{~mW}$. The $\mathrm{THz}$ reflective imaging is performed at almost normal incidence to obtain the data used in the following numerical and experimental study. Prior to carrying out the measurements, the time domain $\mathrm{THz}$ signal (reference signal) produced by the apparatus was recorded by placing a metal plate (i.e. an excellent reflector) at the sample position. The corresponding spectrum of $\mathrm{THz}$ reference signal is obtained by Fourier transforming the pulse. Each recorded reflected THz pulse contains 4096 data points, and the signal is averaged over 10 shots per pixel to reduce the effect of noise.

\section{RESULTS}

Numerical simulation was performed first to verify the performance of $\mathrm{AR} / \mathrm{MCM}$ on multilayered samples. To this end, we consider how AR/MCM performs with synthetic data. An ideal impulse response function $h[n]$, which contains 4096 data points with sampling period $T_{\mathrm{s}}=0.0116 \mathrm{ps}$, is assumed, 


$$
h[n]=\left\{\begin{array}{cl}
0.5 & n=1560 \\
0.5 & n=1620 \\
-0.5 & n=1680 \\
1 & n=1740 \\
0 & \text { otherwise }
\end{array}\right.
$$

where $n$ is the discrete-time variable and $t=n T_{\mathrm{s}}$ and the subscript 0 denotes quantities estimated from synthesized data. $h_{0}[n]$ thus consists of a sequence of four discrete-time impulses, and represents a typical three-layer structure; the time interval between consecutive peaks corresponds to the thickness of each layer. After convolution with the measured reference pulse $f[n]$ from the THz system with the impulse response function $h_{0}[n]$ and adding Gaussian white noise $e_{0}$ uncorrelated with respect to $n$, the reflected $\mathrm{THz}$ signal $r_{0}[n]$ is simulated, as shown in Fig. 4. Based on the experimental dynamic range of the THz system, the signal-to-noise ratio of $r_{0}[n]$ is set to be $50 \mathrm{~dB}$ in the simulation. In the following we shall use the discrete time argument $n$ and the continuous time $t$ interchangeably as convenient.

Because of the high sensitivity of AR to noise, the first step in the procedure, before fitting an AR model, is the estimation of the SNR of the reflected signal. Before implementing $\mathrm{AR} / \mathrm{MCM}$, a wavelet deconvolution procedure is applied, owing to the high similarity of the reference pulse with conveniently selected wavelet basis functions [36]. In contrast to the Fourier transform, which employs an infinite set of sinusoids as the basis, the wavelet transform is an efficient representation of $\mathrm{THz}$ pulse because of the time-frequency localization of the wavelet basis function [37], allowing for a highly truncated wavelet basis to be employed. As a result, wavelet denoising selectively drops noise-like features without utilizing a heavy-handed filter such as a low-pass filter. Therefore, the signal bandwidth after wavelet denoising will not be narrowed. In principle, the wavelet technique decomposes a signal by convolving with a low-pass filter and a high-pass filter at each level and removes the wavelet coefficients with small absolute values by thresholding [38]. Based on Ref. [39], 
balancing the denoising efficiency and computational cost, symlet (sym4) wavelets are selected with a level of 5 for the wavelet decomposition.

Figure 5 presents the comparison of the deconvoluted transfer function (Fourier transform of $h[n]) H_{\text {Inv }}(f)$ by direct inverse filtering and the estimated transfer function by AR/MCM $H_{\mathrm{AR} / \mathrm{MCM}+\text { wavelet }}(f)$ with wavelet denoising. Large spikes due to noise are presented in the lowand high- frequency regions of $H_{\text {Inv }}(f)$. The high-SNR window for the AR model was set to [0.14 THz, $1.75 \mathrm{THz}]$. By applying the data within $[0.14 \mathrm{THz}, 1.32 \mathrm{THz}]$ into the backward prediction filter and the data within $[0.58 \mathrm{THz}, 1.75 \mathrm{THz}]$ into the forward prediction filter, the entire frequency band can be extrapolated. In order to avoid the overfitting, the parameters of the forward and backward prediction filters are controlled by AIC. The criterion is found to reach its minimum when the order $p$ is 70 . By simply performing the inverse Fourier transform of $H_{\mathrm{AR} / \mathrm{MCM}+\text { wavelet }}(f)$, the reconstructed signal $h_{\mathrm{AR} / \mathrm{MCM}+\text { wavelet }}(t)$ can be achieved, as shown in Fig. 6. Compared with the raw reflected signal $r_{0}(t)$ from which it is not obvious how one would visually reconstruct the stratigraphy, $h_{\mathrm{AR} / \mathrm{MCM}+\text { wavelet }}(t)$ exhibits four clear peaks, the exact positions of which are in good agreement with the synthesized impulse response function $h_{0}(t)$, albeit with minor peak shifts as well as the magnitude change, resulting from residual noises even after wavelet denoising as well as the built model. Moreover, the ultimate goal for us are to sharpen the echoes in raw reflected signal and enhance the SNR to improve the temporal resolution, so the amplitude information is not our priority, even though there are also significant. The success of resolving all interfaces demonstrates the potential of $\mathrm{AR} / \mathrm{MCM}$ for characterizing the individual layer of stacked samples.

We next turn to the experimental study of the nine steel coupons. The thickness of each individual layer of all 9 samples are characterized first using high-resolution Deltascope FMP 30, following the deposition of each individual layer, which is a standard and widely used 
approach for measuring coatings on ferrous based materials; However, because the eddycurrent technique cannot resolve the individual layer thicknesses in the complete multilayer coating, the strategy we applied here to obtain the thickness of all individual layers is to perform multiple measurements at different steps in the layer deposition process. Specifically, an Eddy current measurement is performed after the deposition of each layer; simple subtractions can then reveal individual layer thickness. The thicknesses are listed in Table 1. THz TOF tomography is thus carried out following the procedure outlined above, beginning with the steel coupons with a single layer composed of the cataphoretic layer.

\section{A. Single-layer samples}

Figure 7 shows $h_{\mathrm{AR} / \mathrm{MCM}+\text { wavelet }}(t)$ for the single-layer coated samples (a) 5, (b) 9, and (c) 10 . Two positive echoes, corresponding to the air/cataphoretic layer interface, and cataphoretic layer/steel substrate boundaries, are seen in all cases. The cataphoretic-layer thickness is estimated based on the optical delay between the two positive echoes and on the refractive index of cataphoretic material in the THz regime. The optical delay between the first and second positive peaks is (a) $0.14 \mathrm{ps}$, (b) $0.2 \mathrm{ps}$, and (c) $0.25 \mathrm{ps}$, and the refractive index of electrocoat in $\mathrm{THz}$ regime is $\sim 1.72$ [28]. Therefore, the physical thickness of cataphoretic layer on the metal substrate is (a) $12.2 \mu \mathrm{m}$, (b) $17.4 \mu \mathrm{m}$, and (c) $21.8 \mu \mathrm{m}$, respectively. In addition, the uniformity of this layer for samples 5, 9, and 10 are also discussed based on the same 16 pixels that were characterized by the Deltascope (of note, there can be some limited experimental deviation in the pixel positions). The comparison of cataphoretic-layer thickness from Deltascope measurements and AR/MCM for samples 5, 9, and 10 are shown in Fig. 7; see below for discussion. Both the weighted linear fit and $Y=X$ are also plotted. The thickness measured by $\mathrm{AR} / \mathrm{MCM}$ is well correlated with the Deltascope results, confirming the reliability of AR/MCM. To quantify this, the mean square error (MSE) is computed, which is expressed as 


$$
\mathrm{MSE}=\frac{1}{N} \sum_{i=1}^{N}\left(y_{i}-y_{i}^{\prime}\right)^{2}
$$

where $y_{i}$ is the thickness value measured by Deltascope, $y_{i}^{\prime}$ is the thickness value calculated by AR/MCM, and $N$ is the number of data points; here $N$ is 16 . MSE for samples 5,9 , and 10 are is given in Table 2. The thinner the cataphoretic layer, the larger the corresponding MSE, meaning that the error between $\mathrm{AR} / \mathrm{MCM}$ and Deltascope measurements increases as the thickness of cataphoretic layer decreases. The errors result in part from the fact that AR/MCM for the thinner layers may be near its limit of applicability. The thickness of the thinnest cataphoretic layer is close to the minimum time interval that can be distinguished by AR/MCM, which is $\sim 15 T_{s}$ [24]. As the thickness decreases further, the two corresponding peaks in $h_{\mathrm{AR} / \mathrm{MCM}+\text { wavelet }}(t)$ begin to merge, as is seen in Fig. 7(a), and the MSE rises. We have adjusted the parameters of the AR model to separate merged peaks, but the sensitivity of AR model may lead to worse results. In Fig. 8 is shown the cataphoretic-layer thickness reconstructed by $\mathrm{AR} / \mathrm{MCM}$ versus the measured value by Deltascope. We see an overall tendency in the plots for AR/MCM to give slightly higher values than the Deltascope, with this tendency most pronounced for the thinnest layers. The origin of this discrepancy is the partial overlap of the echoes from the air/cataphoretic layer and cataphoretic layer/steel interfaces, which becomes more severe as the cataphoretic-layer thickness decreases.

\section{B. Two-layer samples}

THz TOF tomographic measurements were also carried out on samples 12,14 , and 16 with a cataphoretic layer and sealer on steel, and the corresponding AR/MCM results are presented in Fig. 9. The echo sequence-positive, negative, positive-is seen, corresponding to reflections from the air/sealer, sealer/cataphoretic layer, and cataphoretic layer/steel interfaces, respectively. The negative peak at the sealer/cataphoretic layer interface results from the phase shift of THz signal as the refractive index of the cataphoretic layer is lower than that of 
the sealer. The individual thicknesses of cataphoretic layer and sealer are computed. The assumed refractive index of the sealer is 2.61 [28]. Figure 10 shows the comparison of the cataphoretic layer and sealer thicknesses obtained by Deltascope measurements and AR/MCM. The MSE's for samples 12, 14, and 16 are presented in Table 3. For the sealer, the low MSE demonstrate excellent agreement with the results obtained directly from Deltascope. Somewhat less agreement is seen for the cataphoretic layer, because of the partial overlap of the echoes from the sealer/cataphoretic layer and cataphoretic layer/steel interfaces, leading to uncertainly in the time delay between the echoes. Similar to the remarks above for samples 5, 9, and 10, the error associated with the cataphoretic-layer thickness for samples 12, 14, and 16 increases as the cataphoretic-layer thickness decreases.

\section{Three-layer samples}

We now consider three-layer coatings (lacquer, sealer, cataphoretic layer on steel). Results of AR/MCM are shown in Fig. 11. Four echoes-positive, negative, positive, positivecorresponding to the air/lacquer, lacquer/sealer, sealer/cataphoretic layer, and cataphoretic layer/steel interfaces, respectively, are identified. MSEs of individual layer thicknesses obtained using Deltascope and AR/MCM for samples 11, 15 and 17 are given in Table 4, while Fig. 12 plots the AR/MCM values versus the Deltascope measurements. For the sealer and lacquer, due to their relatively large thicknesses, AR/MCM provides thickness values close to the Deltascope measurements, as the echoes are temporally well separated and distinguishable from the echoes reflected from cataphoretic layer (thin layer). For the cataphoretic layer, again, the greatest deviations between the Deltascope measurements and AR/MCM occur for the thinnest layers.

\section{CONCLUSION}

In summary, THz TOF tomography combined with AR/MCM stratigraphic reconstruction was employed to analyze the thickness of individual layers in multilayer coatings on steel 
with a nondestructive and noncontact fashion. The calculated thickness of each individual layers based on THz-based approach shows a good agreement with eddy-current-based measurements, though deviations are observed when layer thicknesses are $\lesssim 10 \mu \mathrm{m}$. Moreover, $\mathrm{AR} / \mathrm{MCM}$ is a relatively robust analysis technique, that does not require extensive training, nor unlike model-based approaches, does not require a specific physical model to carry our accurate reconstruction.

\section{ACKNOWLEDGEMENTS:}

We gratefully acknowledge the support of by ArcelorMittal Maizières Research SA, Conseil Régional Grand Est, and CPER SusChemProc. 


\section{REFERENCE}

[1]. Babadzhanov LS, Babadzhanova ML. Measurement of the thickness of coatings for use in graduation and calibration of magnetic thickness gauges. Meas. Tech. 1999;42(6):559-62. https://doi.org/10.1007/BF02504413.

[2]. Garicia-Martin J, Gomez-Gil J, Vazquez-Sanchez E. Non-destructive techniques based on eddy current testing, J. Sens. 2011;11(3): 2525-65.https://doi.org/10.3390/s110302525.

[3]. Yin W, Withers PJ, Sharma U, Peyton AJ. Noncontact characterization of carbon-fiberreinforced plastics using multifrequency eddy current sensors. IEEE trans instrum meas. 2008; 58(3): 738-43. 10.1109/TIM.2008.2005072.

[4]. He Y, Tian G,Zhang H, Alamin M, Simm A, Jackson P. Steel corrosion characterization using pulsed eddy current systems. IEEE Sens. J. 2012; 12(6): 211320.10.1109/JSEN.2012.2184280.

[5]. Qin L, Cheng H, Li JM, Wang QM. Characterization of polymer nanocomposite films using quartz thickness shear mode (TSM) acoustic wave sensor. Sens Actuators A Phys. 2007; 136(1): 111-7. https://doi.org/10.1016/j.sna.2006.12.027.

[6]. Pei J, Degertekin FL, Khuri-Yakub BT, Saraswat KC. In situ thin film thickness measurement with acoustic Lamb waves. Appl Phys Lett. 1995; 66(17): 2177-9. $\underline{10.1063 / 1.113938}$.

[7]. Schindel DW, Hutchins DA. Through-thickness characterization of solids by wideband air-coupled ultrasound. Ultrasonics. 1995; 33(1): 11-7. https://doi.org/10.1016/0041$\underline{624 X(95) 00011-Q}$.

[8]. Romero-Torres S, Perez-Ramos JD, Morris KR, Grant ER. Raman spectroscopy for tablet coating thickness quantification and coating characterization in the presence of strong 
fluorescent interference. J. Pharmaceut. Biomed. 2006; 41(3): 811-9. https://doi.org/10.1016/j.jpba.2006.01.033.

[9]. Shen YC. Terahertz pulsed spectroscopy and imaging for pharmaceutical applications: a review. Int. J. $\quad$ pharm. 2011; 417(1-2): 48-60. https://doi.org/10.1016/j.ijpharm.2011.01.012.

[10]. Fukunaga K, Cortes E, Cosentino A, Stunkel I, Leona M, Duling III IN, Miniberg DT. Investigation the use of terahertz pulsed time domain reflection imaging for the study of fabric layers of an Egyptian mummy. J. Eur. Opt. Soc.-Rapid 2011; 6(0); 11040. 10.2971/jeos.2011.11040.

[11]. Fukunaga K, Ogawa Y, Hayashi SI, Hosako I. Terahertz spectroscopy for art conservation. IEICE Electron. Expr. 2007; 4(8): 258-63. https://doi.org/10.1587/elex.4.258.

[12]. Dong J, Locquet A, Citrin DS. Terahertz quantitative nondestructive evaluation of failure modes in polymer-coated steel. IEEE J. Sel. Top. Quant. Electron. 2016; 23(4): 1-7. 10.1109/JSTQE.2016.2611592.

[13]. Choi J, Kwon WS, Kim KS, and Kim S. Nondestructive evaluation of multilayered paint films in ambient atmosphere using terahertz reflection spectroscopy. NDT\&E Int. 2016; 80: 71-6. https://doi.org/10.1016/j.ndteint.2016.02.011.

[14]. Liu HB, Zhong H, Karpowicz N, Chen Y, Zhang XC. Terahertz spectroscopy and imaging for defense and security applications. Proc. IEEE. 2007 ; 95(8): 1514-27. 10.1109/JPROC.2007.898903.

[15]. Zimdars D, White J, Stuk G, Sucha G, Fichter G, Williamson SL. Time domain terahertz imaging of threats in luggage and personnel. Int. J. High-Speed Electron. Syst. 2007; 17(02): 271-81.https://doi.org/10.1142/S0129156407004497. 
[16]. Krimi S, Klier J, Jonuscheit J, von Freymann G, Urbansky R, Beigang R, High accurate thickness of multi-layered automotive paints using terahertz technology. Appl Phys Lett 2016; 109(2): 021105. https://doi.org/10.1063/1.4955407.

[17]. Dong J, Jackson JB, Melis M, Giovanacci D, Walker GC, Locquet A, Bowen JW, Citrin DS. Terahertz frequency-wavelet domain deconvolution for stratigraphic and subsurface investigation of art painting. Opt. Express. 2016; 24(23): 26972-85. https://doi.org/10.1364/OE.24.026972.

[18]. Pickwell E, Wallace VP, Cole BE, Ali S, Longbottom C, Lynch RJ, Pepper M, A comparison of terahertz pulsed imaging with transmission microradiography for depth measurement of enamel demineralization in virto. Caries Res 2007; 41(1): 49-55. https://doi.org/10.1159/000096105.

[19]. Woodward RM, Cole BE, Wallace VP, Pye RJ, Arnone DD, Linfield EH, Pepper M. Terahertz pulse imaging in reflection geometry of human skin cancer and skin tissue. Phys. Med 2002; 47(21):3853. https://doi.org/10.1088\%2F0031-9155\%2F47\%2F21\%2F325

[20]. Chen Y, Huang S, Pickwell-MacPherson E. Frequency-wavelet domain deconvolution for terahertz reflection imaging and spectroscopy. Opt. Express 2010; 18(2): 1177-90. https://doi.org/10.1364/OE.18.001177.

[21]. Hu W, Wang W, Ji J, Si L. The spatial resolution enhancement deconvolution technique of the optimized wiener filter in Terahertz band. In 2016 IEEE $9^{\text {th }}$ UK-Europe-China Workshop on Millimetre Waves and Terahertz Techniques (UCMMT) (pp. 96-99). IEEE. 10.1109/UCMMT.2016.7873975.

[22]. Dong J, Locquet A, Citrin DS. Terahertz super-resolution stratigraphic characterization of multilayered structures using sparse deconvolution. IEEE Trans. Terahertz Sci. Techn. 2017; 7(3): 260-7.10.1109/TTHZ.2017.2673542. 
[23]. Dong J, Locquet A, Melis M, Citrin DS. Global mapping of stratigraphy of an old-master painting using sparsity-based terahertz reflectometry. Sci. Rep 2017; 7(1): 1-12. https://doi.org/10.1038/s41598-017-15069-2.

[24]. Dong J, Locquet A, Citrin DS. Depth resolution enhancement of terahertz deconvolution by autoregressive spectral extrapolation. Opt. Lett. 2017; 42(9): 1828-31. https://doi.org/10.1364/OL.42.001828.

[25]. Chang T, Guo Q, Liu L, Cui HL. Hilbert-transform-based accurate determination of ultrashort-time delays in terahertz time-domain spectroscopy. IEEE Trans. Terahertz Sci. Techn. 2017; 7(5): 514-20. 10.1109/TTHZ.2017.2713602.

[26]. Yasui T, Yasuda T, Sawanaka KI, Araki T. Terahertz paintmeter for noncontact monitoring of thickness and drying progress in paint film. Appl. Opt 2005; 44(32): 684956. https://doi.org/10.1364/AO.44.006849.

[27]. Yasuda T, Iwata T, Araki T, Yasui T. Improvement of minimum paint film thickness for THz paint meters by multiple-regression analysis, Appl. Opt 2007;46(30): 7518-26. https://doi.org/10.1364/AO.46.007518.

[28]. Su K, Shen YC, Zeitler JA. Terahertz sensor for non-contract thickness and quality measurement of automobile paints of varying complexity. IEEE Trans. Terahertz Sci. Techn. 2014; 4(4): 432-9. 10.1109/TTHZ.2014.2325393.

[29]. Krimi S, Klier J, Herrmann M, Jonuscheit J, Beigang R. Inline multilayer thickness sensing by using terahertz time-domain spectroscopy in reflection geometry. In2013 $38^{\text {th }}$ International Conference on Infrared, Millimeter, and Terahertz Waves (IRMMW-THz) 2013 Sep 1 (pp. 1-2). IEEE. 10.1109/IRMMW-THz.2013.6665871.

[30]. Ellrich F, Klier J, Weber S, Jonuscheit J, von Freymann G. Terahertz time-domain technology for thickness determination of industrial relevant multi-layer coatings. In2016 
$41^{\text {st }}$ International Conference on Infrared, Millimeter, and Terahertz waves (IRMMWTHz) 2016 Sep 25 (pp. 1-2). IEEE. 10.1109/IRMMW-THz.2016.7758614.

[31]. Lines LR, Clayton RW. A new approach to vibroiseis deconvolution. Geophys. Prospect 1977; 25(3): 417-33. https://doi.org/10.1111/j.1365-2478.1977.tb01179.x.

[32]. Akaike H. Information theory and an extension of the maximum likelihood principle. In Selected papers of hirotugu Akaike. Springer, New York, NY; 1998.

[33]. Alessio SM. Digital signal processing and spectral analysis for scientists: Concepts and application. Springer; 2015.

[34]. Shakibi B, Honarvar F, Moles MD, Caldwell J, Sinclair AN. Resolution enhancement of ultrasonic defects signals for crack sizing. NDT\&E Int. 2012; 52: 37-50. https://doi.org/10.1016/j.ndteint.2012.08.003.

[35]. Gupta IJ, Beals MJ, Moghaddar A. Data extrapolation for high resolution radar imaging. IEEE Trans. Antennas and Propag. 1994;42(11):1540-5. 10.1109/8.362783.

[36]. Kazubek M. Wavelet domain imaging denoising by thresholding and wiener filtering. IEEE Signal Proc. Lett. 2003; 10(11): 324-6. 10.1109/LSP.2003.818225.

[37]. Mittleman DM, Jacobsen RH, Nuss MC. T-ray imaging. IEEE J. Sel. Top. Quant. Electron. 1996; 2(3): 679-92. 10.1109/2944.571768.

[38]. Mittleman DM, Jacobsen RH, Neelamani R, Baraniuk RG, Nuss MC. Gas sensing using terahertz time-domain spectroscopy. Appl. Phys. B 1998; 67(3): 379-90. http://dx.doi.org/10.1007/s003400050520.

[39]. Pei J, Ye P, Xie W. Optimal wavelet analysis for THz-TDS pulse signals. Proc of SPIE 2009;7277: 727708.10.1117/12.819905. 


\section{FIGURE}

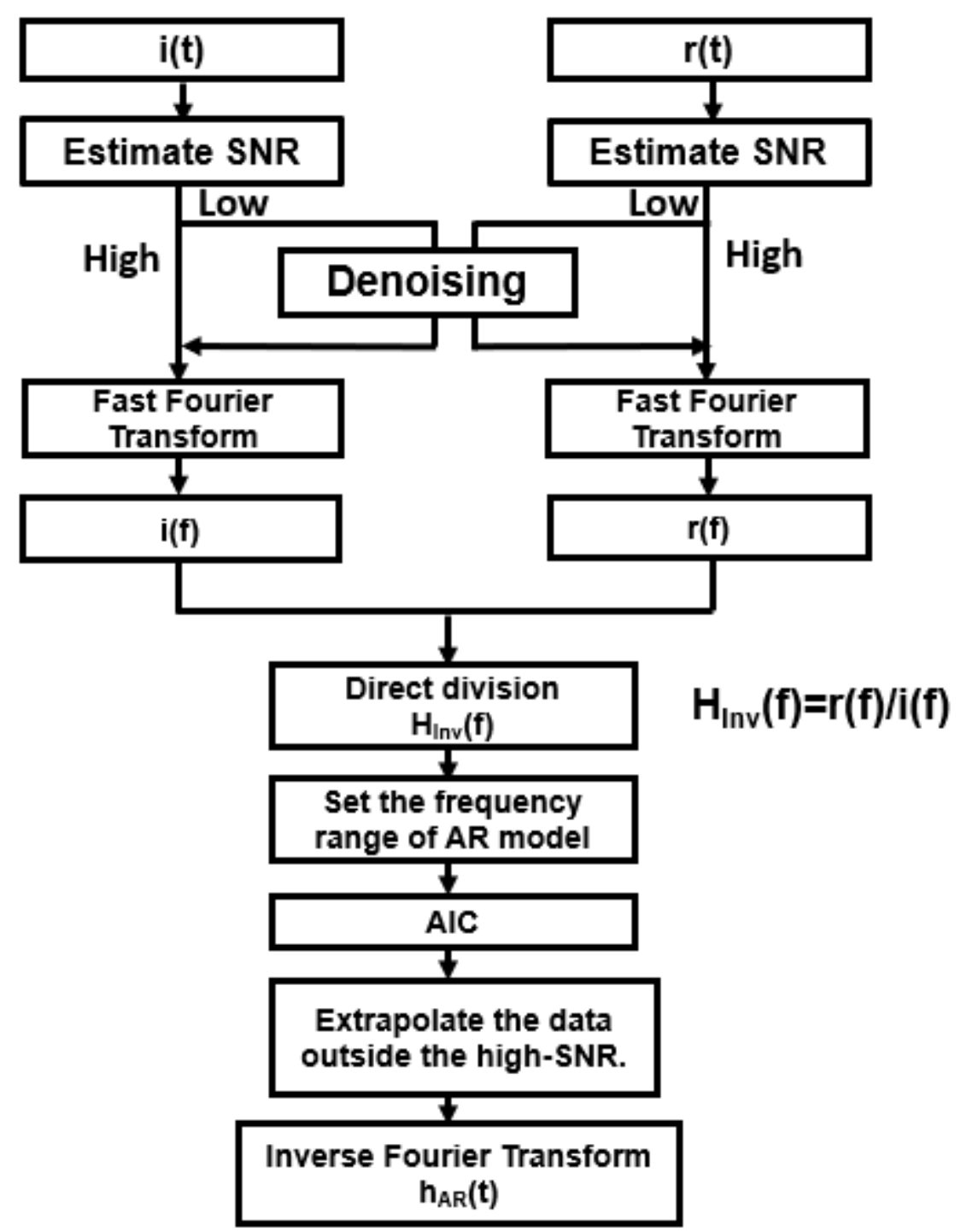

Fig. 1. Block diagram of the application of the AR deconvolution algorithm. 


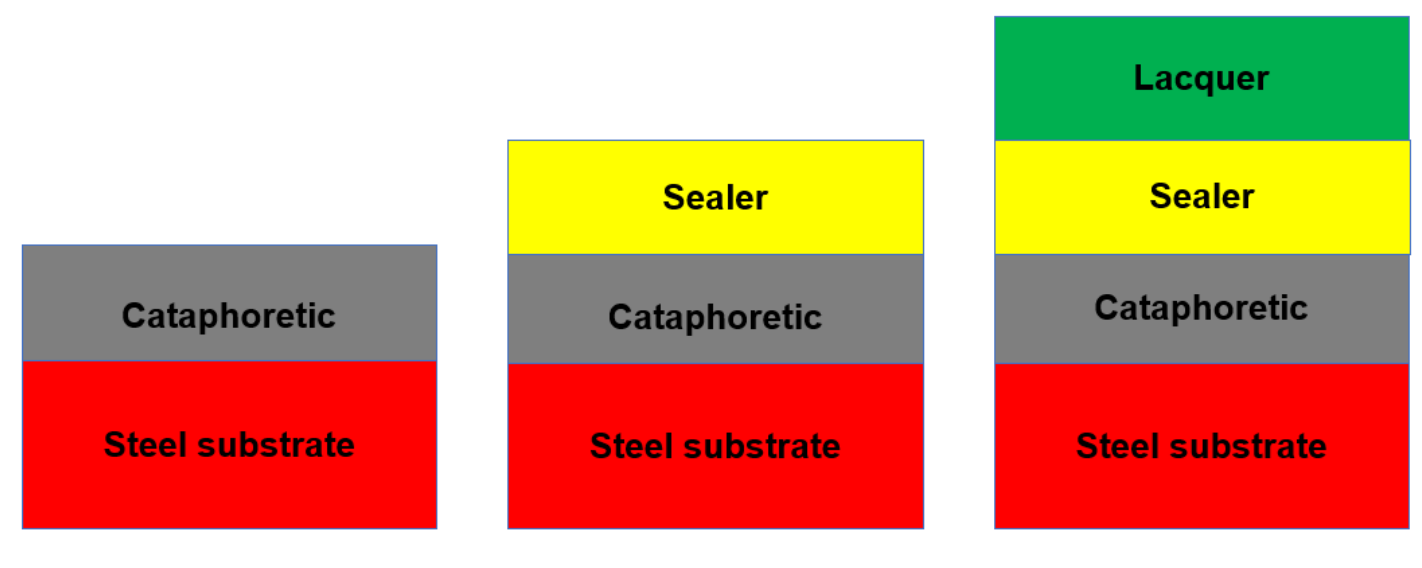

Fig. 2 The cross-sectional schematic diagrams of the multi-layered samples in this study. 


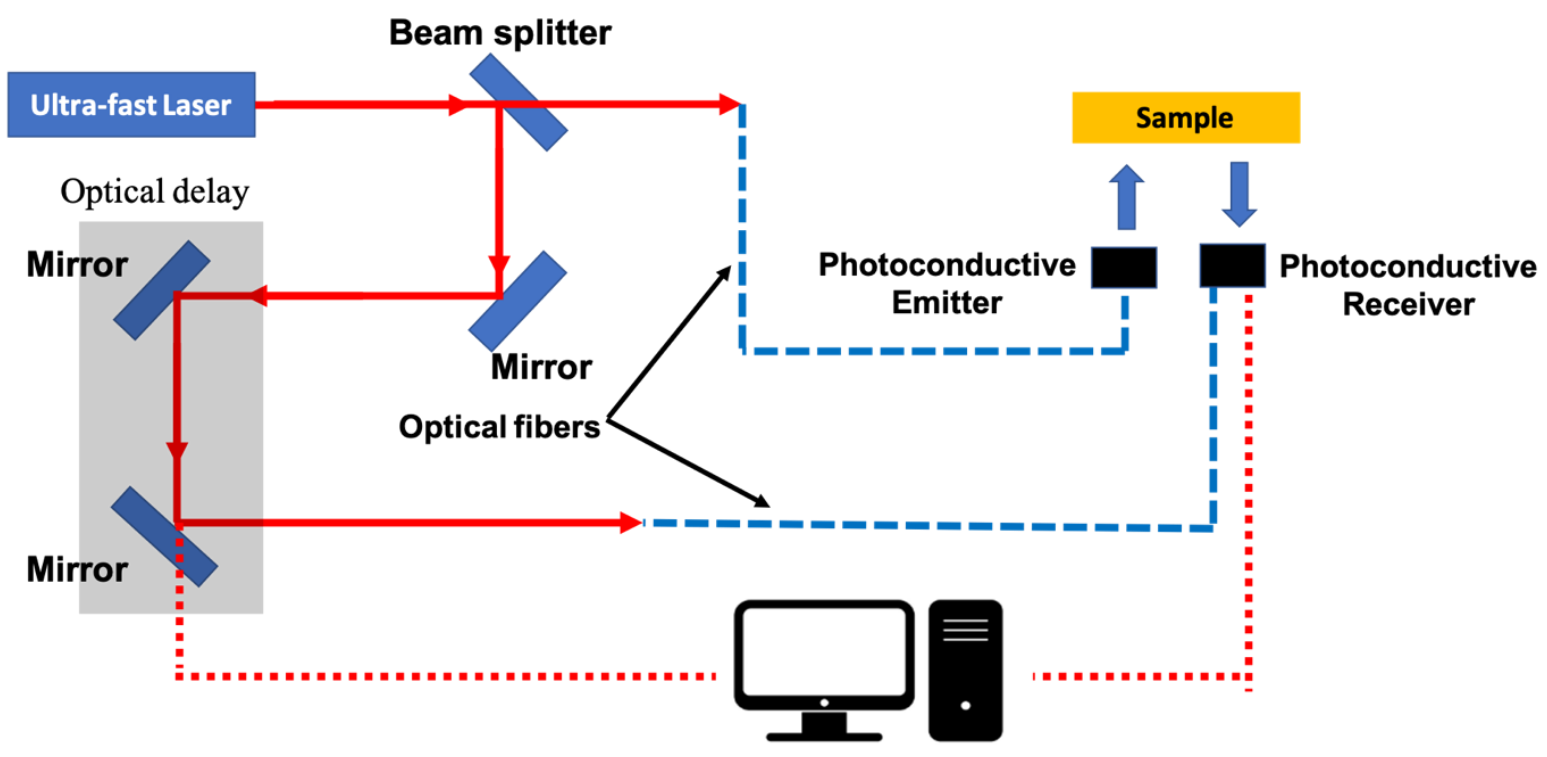

Fig. 3 Schematic diagram of the THz TOF tomography system. 


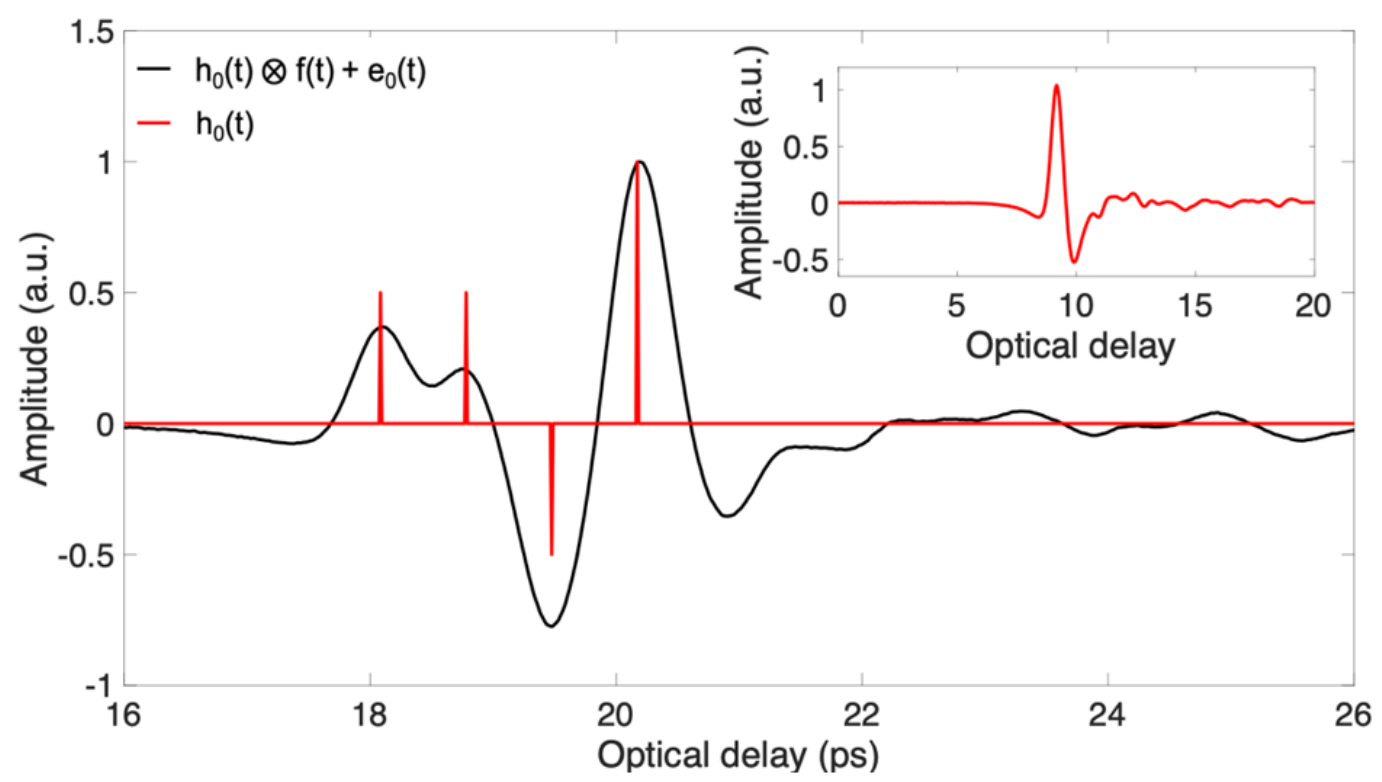

Fig. 4 Synthetic impulse response function $h_{0}(t)$ and the simulated reflected $\mathrm{THz}$ signal $h_{0}(t) \otimes f(t)+e_{0}(t)$. The inset shows the experimental reference signal $f(t)$. 


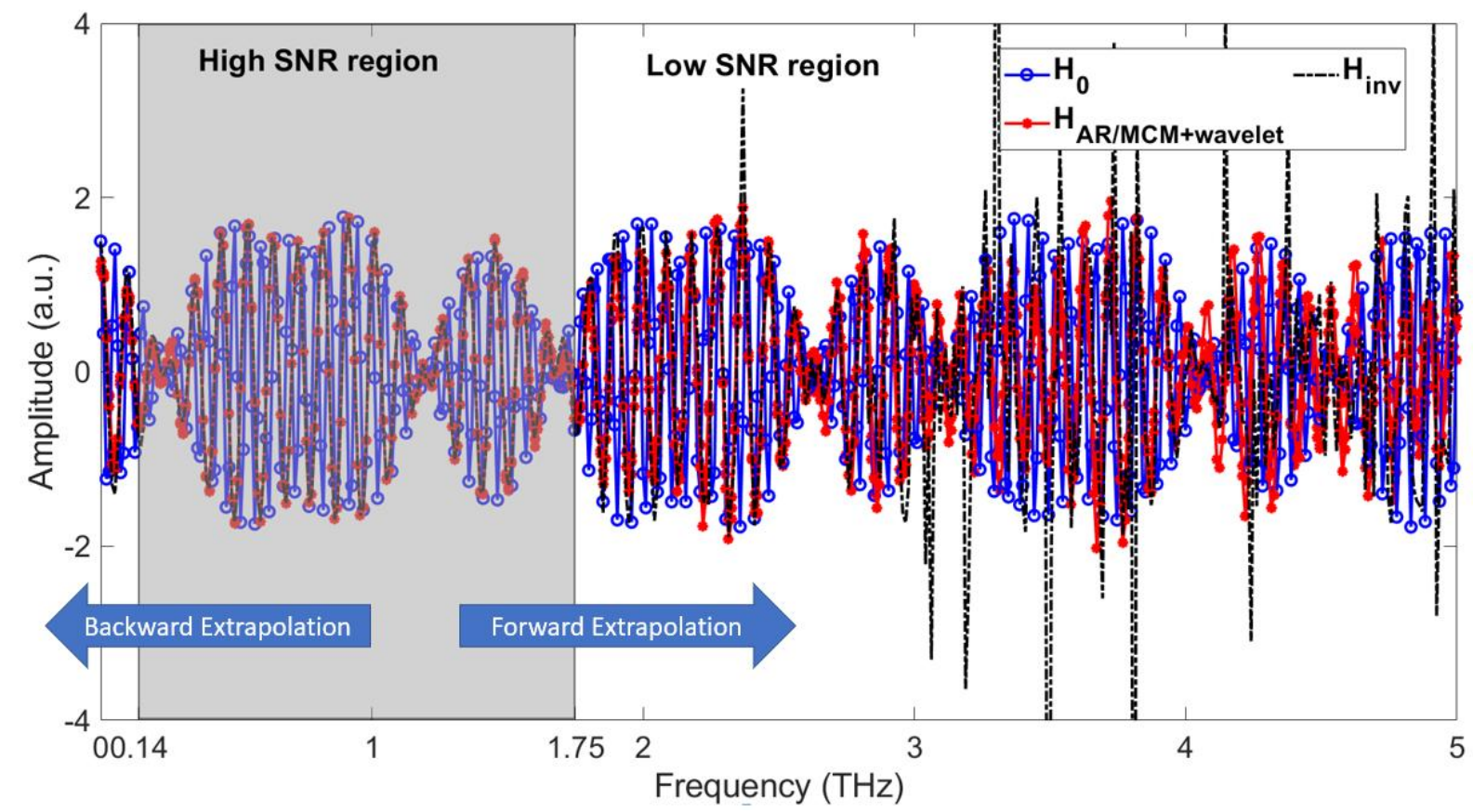

Fig. 5 Comparison between the deconvoluted spectrum by direct inverse filtering $H_{\text {Inv }}(f)$ (dashed black) and the estimated frequency spectrum by (red) AR/MCM with wavelet denoising. $H_{0}$ (blue) is the transfer function obtained by Fourier transforming the model impulse response function $h_{0}(t)$. 


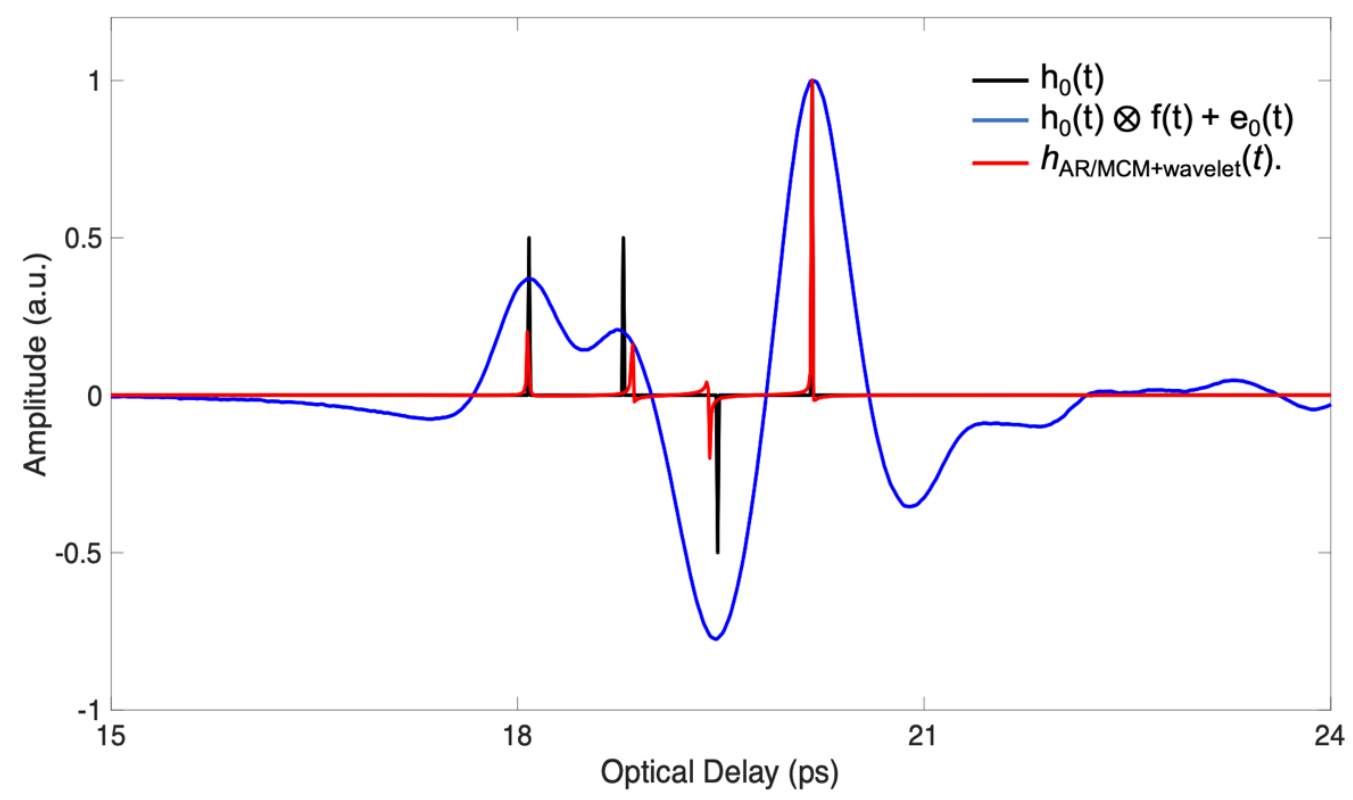

Fig. 6 Comparison of the simulated deconvolution results $h_{\mathrm{AR} / \mathrm{MCM}+\mathrm{wavelet}}(t)$ based on $\mathrm{AR} / \mathrm{MCM}$ (red), the raw reflected signal $h_{0}(t) \otimes f(t)+e_{0}(t)$ (blue), and the assumed impulse response function $h_{0}(t)$ (black). 

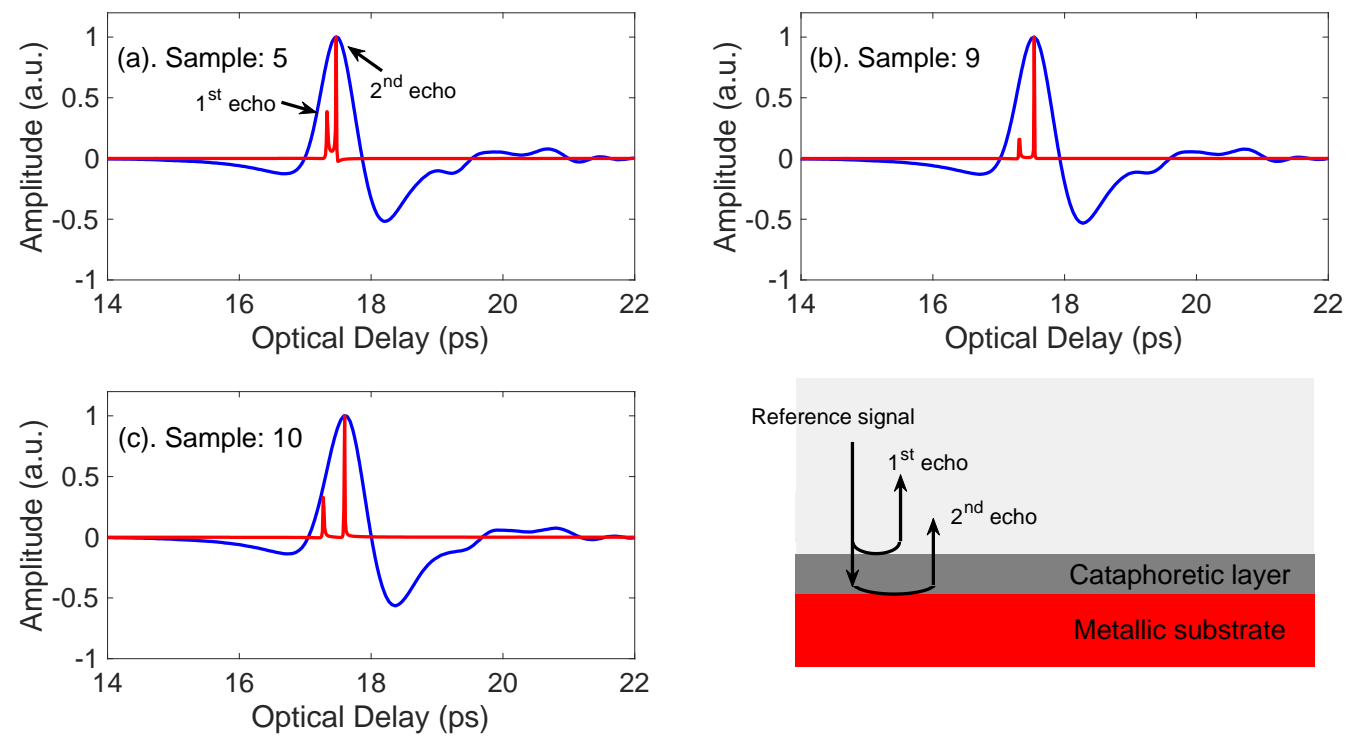

Fig. 7 The AR/MCM result for samples (a) 5, (b) 9, and (c) 10. 

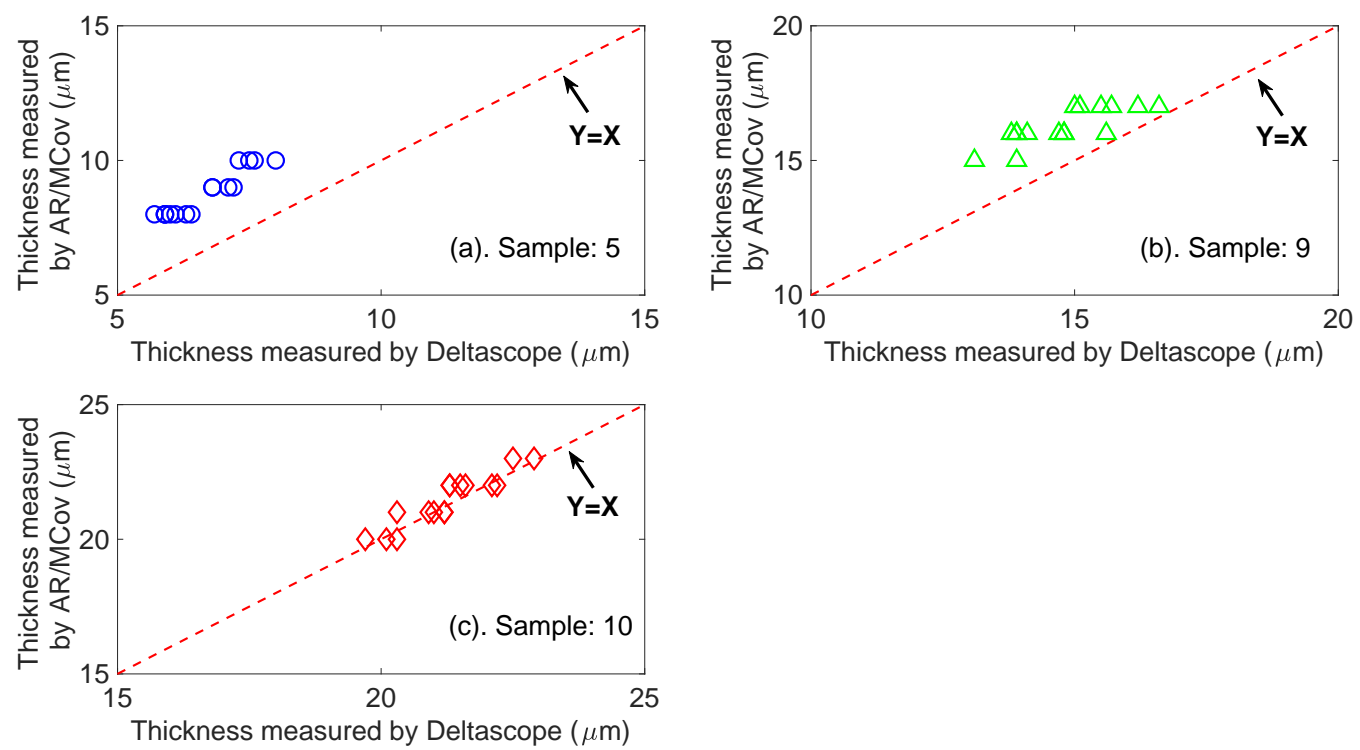

Fig. 8 Thickness of cataphoretic layer measured by AR/MCM method as a function of thickness measured by Deltascope for samples (a) 5, (b) 9, and (c) 10. 

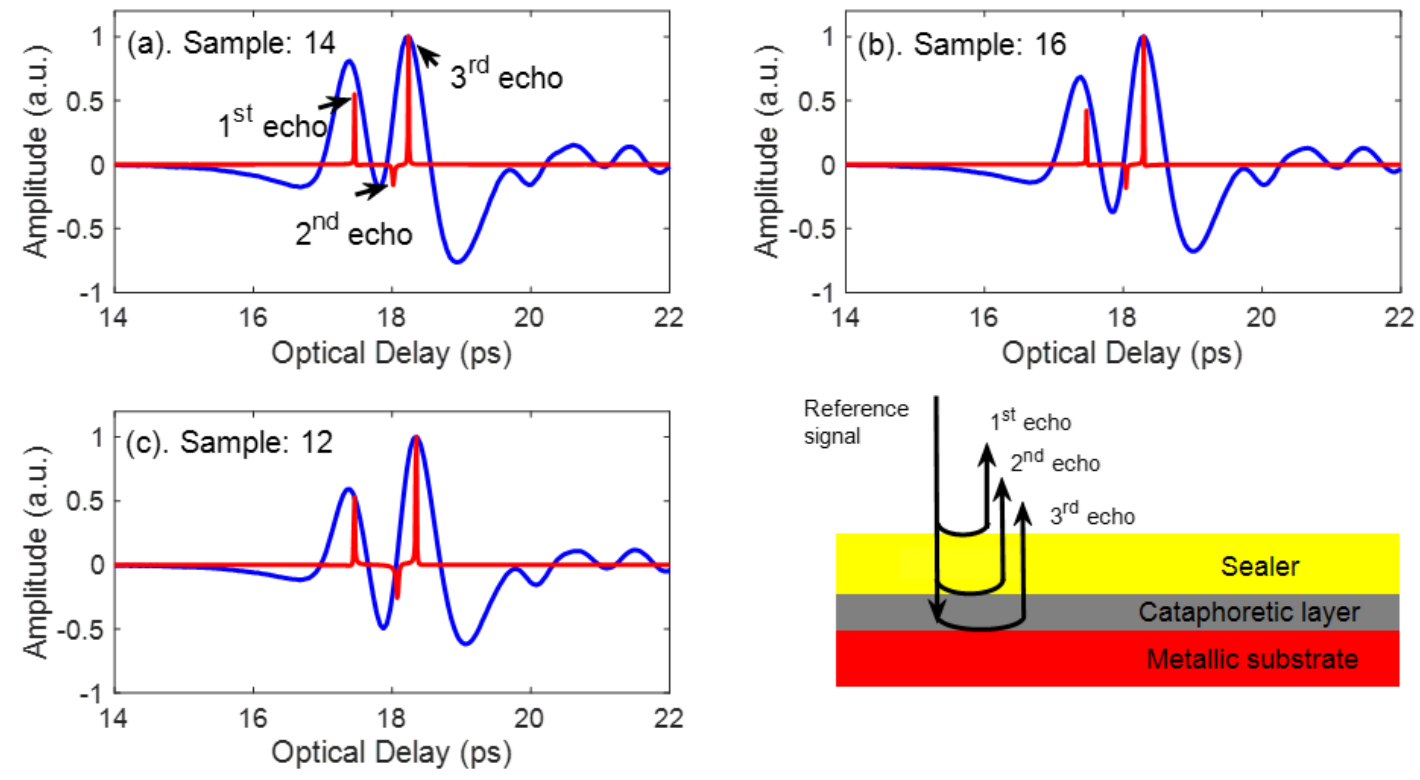

Fig. 9 The AR/MCM result of samples (a) 14, (b) 16, and (c) 12. 

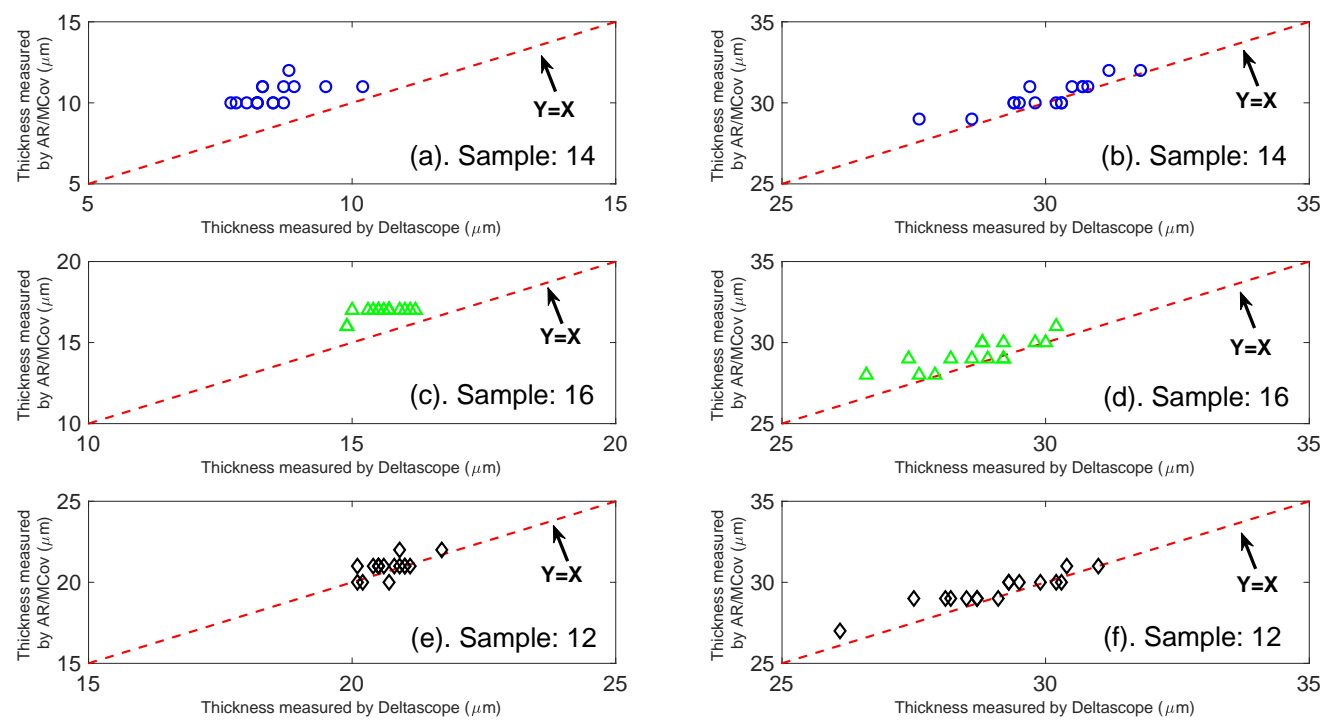

Fig. 10 Comparison of the thickness of individual layers for samples 12, 14, and 16 between AR/MCM and the Deltascope. Left column: thickness of cataphoretic layer as a function of thickness measured by Deltascope; Right column: thickness of sealer as a function of thickness measured by Deltascope. 

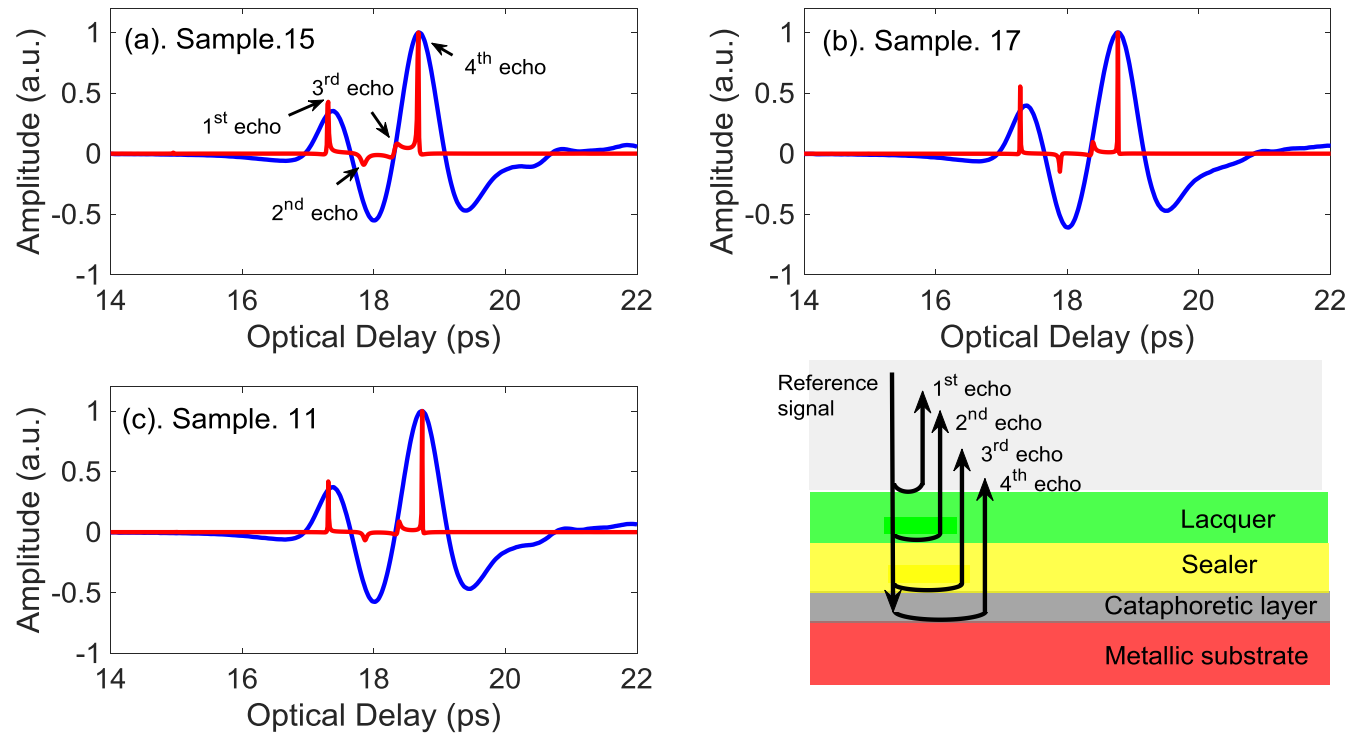

Fig. 11 The AR/MCM result of samples (a) 15, (b) 17, and (c) 11. 

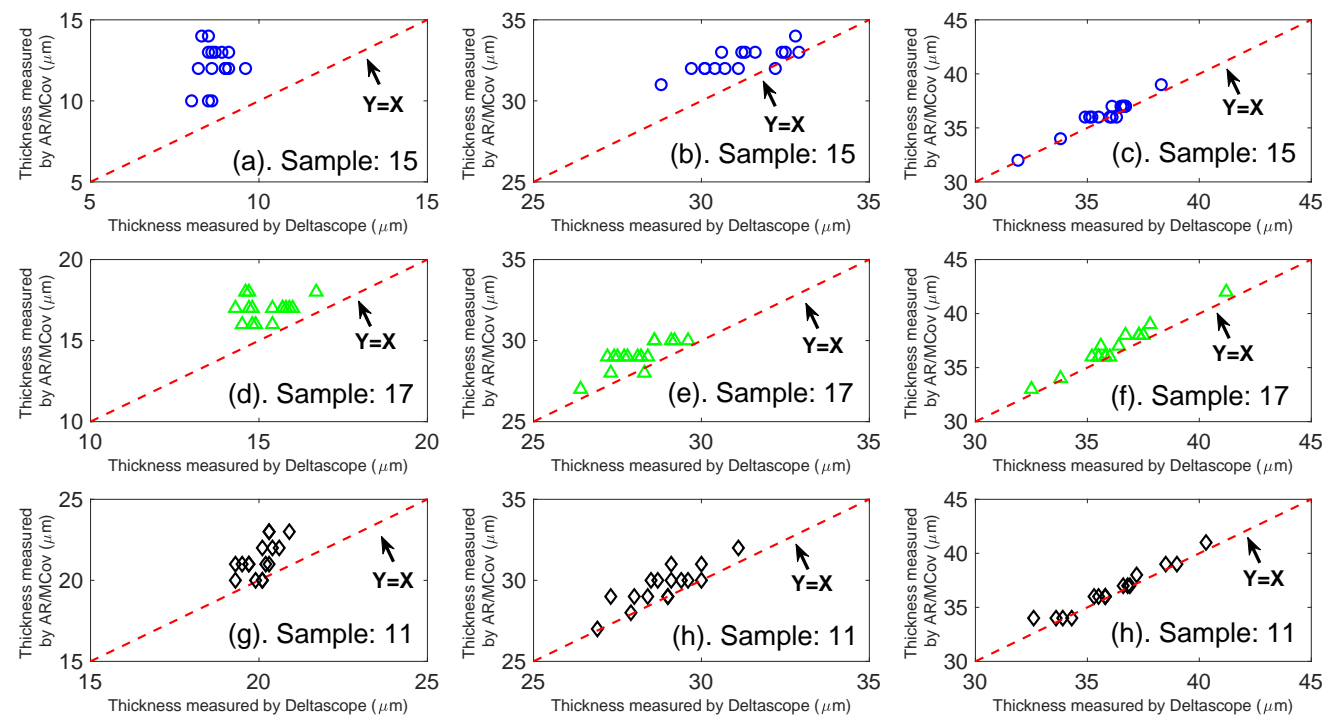

Fig. 12 Comparison of the thickness of individual layers for samples 11, 15, and 17 between AR/MCM and Deltascope. Left column: thickness of cataphoretic layer as a function of thickness measured by Deltascope for samples 11, 15, 17; Middle column: thickness of sealer as a function of thickness measured by Deltascope for samples 11, 15, and 17; Right column: thickness of lacquer as a function of thickness measured by Deltascope for samples 11, 15, and 17. 


\section{TABLE}

Table 1 Mean thicknesses $\mu$ and standard deviations $\sigma$ of cataphoretic layer, sealer, and lacquer for all samples obtained via Deltascope measurements.

\begin{tabular}{c|c|c|c|c|c|c}
\hline \multirow{2}{*}{ Sample } & \multicolumn{2}{c|}{ Cataphoretic layer } & \multicolumn{2}{c|}{ Sealer } & \multicolumn{2}{c}{ Lacquer } \\
\cline { 2 - 7 } & $\mu(\mu \mathrm{m})$ & $\sigma(\mu \mathrm{m})$ & $\mu(\mu \mathrm{m})$ & $\sigma(\mu \mathrm{m})$ & $\mu(\mu \mathrm{m})$ & $\sigma(\mu \mathrm{m})$ \\
\hline 5 & 6.6 & 0.7 & & & & \\
9 & 14.8 & 1 & & & & \\
10 & 21.3 & 0.9 & & & & \\
16 & 15.6 & 0.4 & 28.4 & 1.4 & & \\
12 & 20.7 & 0.4 & 29 & 1.2 & & \\
14 & 8.5 & 0.6 & 30 & 1 & & \\
15 & 8.7 & 0.4 & 31.1 & 1.2 & 35.8 & 1.4 \\
11 & 20 & 0.5 & 28.9 & 1.1 & 36.1 & 2 \\
17 & 15.2 & 0.7 & 28.1 & 0.8 & 36.3 & 1.9 \\
\hline
\end{tabular}


Table 2 MSEs of the thickness of cataphoretic layer based on the Deltascope and AR/MCM for samples 5, 9, and 10 .

\begin{tabular}{c|c}
\hline Sample & $\begin{array}{c}\text { MSE of cataphoretic layer } \\
\left(\boldsymbol{\mu m}^{2}\right)\end{array}$ \\
\hline 5 & 8.4 \\
9 & 2.4 \\
10 & 2.4 \\
\hline
\end{tabular}


Table 3 MSEs of the thickness of cataphoretic layer and sealer based on and Deltascope and AR/MCM for samples 12, 14, and 16.

\begin{tabular}{c|c|c}
\hline Sample & $\begin{array}{c}\text { MSE of cataphoretic layer } \\
\left(\boldsymbol{\mu m}^{\mathbf{2}}\right)\end{array}$ & $\begin{array}{c}\text { MSE of sealer } \\
\left(\boldsymbol{\mu \mathbf { m }}^{\mathbf{2}}\right)\end{array}$ \\
\hline 12 & 1.2 & 0.4 \\
14 & 4.2 & 0.4 \\
16 & 1.8 & 0.7 \\
\hline
\end{tabular}


Table 4 MSEs of the thickness of the cataphoretic layer, sealer, and lacquer for samples 11,15, and 17 .

\begin{tabular}{c|c|c|c}
\hline Sample & $\begin{array}{c}\text { MSE of cataphoretic layer } \\
\left(\boldsymbol{\mu \mathbf { m } ^ { 2 } )}\right.\end{array}$ & $\begin{array}{c}\text { MSE of sealer } \\
\left(\boldsymbol{\mu m}^{\mathbf{2}}\right)\end{array}$ & $\begin{array}{c}\text { MSE of lacquer } \\
\left(\boldsymbol{\mu \mathbf { m } ^ { 2 } )}\right.\end{array}$ \\
\hline 11 & 2.3 & 0.9 & 0.3 \\
15 & 13.7 & 2.4 & 0.3 \\
17 & 3.5 & 1.2 & 0.6 \\
\hline
\end{tabular}

